\title{
Modulated Soliton Solution of the Modified Kuramoto-Sivashinsky's Equation
}

\author{
Clovis Taki Dje umen Tchaho ${ }^{1}$, Jean Roger Bogning ${ }^{2, *}$, Timoléon Crépin Kofané ${ }^{1}$ \\ ${ }^{1}$ Department of Phy sics, Faculty of Science, University of Yaoundé I, PO Box 812, Yaoundé, Cameroon \\ ${ }^{2}$ Department of Phy sics, Higher Teacher's Training College, University of Bamenda, PO Box 39, Bamenda, Cameroon
}

\begin{abstract}
We constructed in this work a modulated soliton solution. This solution is a multiform soliton prototype modulated by the very small parameter $\varepsilon$. In practice it can represent, a model of soliton capable of changing its form with respect to the obstacle in its medium of propagation without any loss in its initial energy. The nonlinear partial differential equation used in the construction of this solution is that of modified Kuramoto-Sivashinsky.
\end{abstract}

Keywords Kuramoto-Sivashinsky, Soliton Solution, BDK Method, Kink, Pulse, Multiform Soliton

\section{Introduction}

All physical phenomena are in most cases governed by differential equations and especially by the nonlinear partial differential equations (NPDE) for most complicated cases. Among these NPDEs, the most regular are that of S chröding er, Ginzburg-Land au, Kd V and Kuramoto-Sivashinsky $[1-4]$. The essential thing is not to obtain of NPDEs, but to propose possible solutions. It is in this light that many resolution techniques have been proposed $[5-32]$. In our recent works we proposed a new method of construction of solutions of NPDEs named the method of identification of coefficients of hyperbolic functions or Bogning-Djeumen Tchaho-Kofané method $(\mathrm{BDKm})[33-36]$. In this work, we use the BDKm to construct the soliton solutions formed by combining solutions of type kink and pulse according to the degree of dominion of the parameter $\varepsilon$. It is necessary to say that here we are enlivened by the desire to construct a type of solitary wave solution that is the combination of several shapes of solitary waves. If we can already confess that on the mathematical plan it is possible, on the other hand we cannot already say explicitly what such a solution can represent in the practice or in the physics in general. While we think that in the case where the broadcast of such a signal would be possible in practice, it would be a solitary wave that will be able to change shape according to the characteristic properties of the propagation medium or merely of the met obstacle. To reach our goal, we needed a differential

* Corresponding author:

rbogning@yahoo.com (Jean Roger Bogning)

Published online at http://journal.sapub.org/ajcam

Copyright (C 2012 Scientific \& Academic Publishing. All Rights Reserved equation where cohabits the scattering and the non linearity. For this reason we chose a differential equation presenting a very strong non linearity. Thus, the construction of this multiform solution is supported by Kuramoto-Sivashinsky's equation. This method has been chosen due to the fact that it is more adapted for the construction or amelioration of soliton solution in NPDEs.

This work is organized as follows:

In section 2, we are going to present the BDK method and in section 3 construct or propose a solution which is closer to the form envisaged. In section 4 we will polish up our work.

\section{The BDK Method}

This method is based on the construction of soliton solutions of certain types of nonlinear equations of the form $[33-36]$

$$
\begin{aligned}
& \gamma_{i} \sum_{i}\left(\frac{\partial u}{\partial x_{i}}\right)+b_{i} \sum_{i} \frac{\partial^{2} u}{\partial x_{i}^{2}}+\ldots+c_{i} \sum_{i} \frac{\partial^{l} u}{\partial x_{i}^{l}} \\
& +d_{i} \sum_{n, m} \frac{\partial^{n} u \partial^{m} u}{\partial x_{i}^{l} \partial x_{i}^{m}}+f\left(u,|u|^{2}\right)=0
\end{aligned}
$$

where $\gamma_{i}, b_{i}, c_{i}, d_{i}$ are constants, $f$ a linear function of $u$ and $|u|^{2}$ and $u$ the variable to determine. Knowing that majority of soliton solutions have their analytic forms constituted by functions exp, sinh, cosh, arctan , tanh, sec $h, \cos e c h . .$, we have imagined the form of solutions capable of bringing together the different functions seen above. Among all general forms of solutions which have come across our minds, the most adapted is

$$
u=a_{i j} \sinh ^{j} \alpha x \sec h^{i} \alpha x,
$$


where $a_{i j}$ are constants to determine, $\alpha$ considered as known constant, $i$ and $j$ are natural integers. Why have we chosen solution (Eq.(2))? Simply because it covers effectively the majority of soliton solutions that we come across depending on the variation of integers $i$ and $j$.

The methodic principle is based on determining the constants $a_{i j}$. If we propose the construction of solutions of Eq.(1) in the form

$$
u=\sum_{i j} a_{i j} \sinh ^{j} \alpha x \sec h^{i} \alpha x,
$$

taking into account Eq.(3) in Eq.(1) we get an equation of the form indicated below

$\sum_{i, j, n} F\left(a_{i j}\right) \sec h^{n} \alpha x+\sum_{i, j, m} G\left(a_{i j}\right) \sinh \alpha x \sec h^{m} \alpha x$

$+\sum_{i, j, k} H\left(a_{i j}\right) \cosh ^{k} \alpha x+\sum_{i, j, l} T\left(a_{i j}\right) \cosh ^{l} \alpha x \sinh \alpha x$

$+\sum_{i, j} W\left(a_{i j}\right)=0$

In the relation given by Eq.(4), the factors $\sec h^{n} \alpha x$, $\sinh \alpha x \operatorname{sech}^{m} \alpha x \quad, \quad \cosh ^{k} \alpha x \quad$ and $\cosh ^{l} \alpha x \sinh \alpha x$ are considered as a sort of simple elements of Eq.(4). We wish simply to mention the fact that on introducing Eq.(3) in Eq.(1) we obtain hyperbolic functions which are not directly simple elements obtained in Eq.(4). To come back to the form Eq.(4), it is necessary to use adequate transformations $[33-36]$.On identifying the different coefficients of simple elements of Eq.(4) to zero, we obtain a range of equations in $a_{i j}$ to determine. In all these equations those which have exact values and those which have values more or less closes to exact values respect a certain order of priority ( high value of $n$ and $m$ ). It is convenient to mention here that the choice of the solution to be made is fundamental. When the choice is not appropriate, the results will be contradictory.

In the lines that follow we will go beyond the classical ways of calculation as seen in our previous works $[33-36]$, construction of modulated soliton solution by the parameter $\varepsilon$.

\section{Solutions of the Shape}

$$
\psi=a \sec h^{2} \alpha \xi+b \tanh \alpha \xi+\varepsilon\left(\beta \sec h^{4} \alpha \xi+\gamma \tanh ^{2} \alpha \xi\right)
$$

The modified Kuramoto-Sivashinsky's equation is given by $[33]$

$$
\begin{aligned}
& c \psi^{\prime}+L_{1} \psi^{\prime \prime}+L_{2} \psi^{\prime 2}+L_{3} \psi^{\prime} \psi^{\prime \prime \prime}+L_{4} \psi^{\prime \prime \prime \prime} \\
& +L_{5} \psi^{\prime 2} \psi^{\prime \prime}+L_{6} \psi^{\prime \prime 2}=0,
\end{aligned}
$$

where $\psi(x, t)=\psi(\xi), \xi=x-c t, \psi^{\prime}, \psi^{\prime \prime}, \psi^{\prime \prime \prime}$ and $\psi^{\prime \prime \prime \prime}$ represent respectively the first derivative, the second derivative, the third derivative and the fourth derivative of $\psi$ with respect to $\xi, L_{v}$ with $v=1,2,3,4,5,6$ are the constants, $c$ the group velocity.

The solutions of the shape

$$
\begin{aligned}
& \psi(\xi)=a \sec h^{2} \alpha \xi+b \tanh \alpha \xi \\
& +\varepsilon\left(\beta \sec h^{4} \alpha \xi+\gamma \tanh ^{2} \alpha \xi\right)
\end{aligned}
$$

Introduced in Eq.(5) leads to the relation

$$
\begin{aligned}
& \alpha\left(c b+4 \alpha L_{2} a^{2}\right) \sec h^{2} \alpha \xi \\
+ & \alpha^{2}\left(L_{2} b^{2}+\alpha^{2} L_{6} b^{2}+4 \alpha^{2} L_{3} b^{2}-2 L_{1} a\right) \sec ^{4} \alpha \xi \\
+ & 2 \alpha^{2}\left(\begin{array}{l}
4 \alpha^{2} L_{5} a b^{2}-4 \alpha^{2} L_{5} a^{3} \\
-2 \alpha^{2} L_{6} b^{2}-2 a^{2} L_{2}-3 \alpha^{2} L_{3} b^{2}
\end{array}\right) \sec h^{6} \alpha \xi \\
+ & 2 \alpha^{4} L_{3} a b \sec h^{7} \alpha \xi \\
+ & 2 \alpha^{4} a\left(2 L_{6} a-5 L_{5} b^{2}-40 \beta L_{5} a\right) \sec h^{8} \alpha \xi
\end{aligned}
$$

$+8 \alpha^{4} a^{3} L_{5} \sec h^{10} \alpha \xi+80 \alpha^{4} a^{2} \beta L_{5} \sec h^{12} \alpha \xi$

$-2 \alpha\left(c a+\left(\alpha L_{1}+4 \alpha^{3} L_{4}\right) b\right) \sinh \alpha \xi \sec h^{3} \alpha \xi$

$+4 \alpha^{2}\left(\begin{array}{l}2 \alpha^{2} L_{4}-L_{2} a-2 \alpha^{2} L_{3} a \\ -2 \alpha^{2} L_{5} a^{2}\end{array}\right) b \sinh \alpha \xi \sec h^{5} \alpha \xi$

$-40 \alpha^{4} L_{4} a \sinh \alpha \xi \sec h^{6} \alpha \xi$

$+2 \alpha^{4}\left[\left(6 L_{3}+4 L_{6}\right) a b-L_{5} b^{3}\right] \sinh \alpha \xi \sec h^{7} \alpha \xi$

$-16 a^{2} \alpha^{4} L_{3} \sinh \alpha \xi \sec h^{8} \alpha \xi$

$$
+\varepsilon\left\{+2 \alpha^{2}\left[\begin{array}{c}
3\left(L_{1}+20 \alpha^{2} L_{4}\right) \gamma \\
+8\left(L_{1}+16 \alpha^{2} L_{4}\right) \beta \\
-4\left(L_{2}+2 \alpha^{2} L_{3}\right) \gamma a \\
-8 \alpha^{2} L_{5} a^{2} \gamma
\end{array}\right] \sec h^{4} \alpha \xi\right.
$$$$
-4 \alpha^{2} \gamma\left(L_{1}+4 \alpha^{2} L_{4}\right) \sec h^{2} \alpha \xi
$$

$$
+4 \alpha^{2}\left[\begin{array}{l}
\alpha^{2} L_{5}\left(6 \gamma a^{2}+16 \beta a^{2}-3 \gamma b^{2}\right) \\
+4\left(L_{2}+8 \alpha^{2} L_{3}\right) \beta a \\
+2\left(L_{2}+8 \alpha^{2} L_{3}\right) \gamma a \\
-5\left(L_{1}+52 \alpha^{2} L_{4}\right) \beta \\
-30 \alpha^{2} L_{4} \gamma
\end{array}\right] \sec h^{6} \alpha \xi
$$




$$
\begin{array}{r}
+2 \alpha^{2}\left[\begin{array}{l}
420 \alpha^{2} L_{4} \beta a \\
-8\left(L_{2}+23 \alpha^{2} L_{3}+4 \alpha^{2} L_{6}\right) \beta a \\
-3 \alpha^{2}\left(2 L_{3}+L_{6}\right) \gamma a \\
+\alpha^{2} L_{5}\left(16 \gamma a^{2}+16 \beta b^{2}+3 \gamma b^{2}\right)
\end{array}\right] \sec h^{8} \alpha \xi \\
+16 \alpha^{4}\left[\begin{array}{l}
9 L_{3}-2 L_{5}(\gamma+2 \beta) b a+5 L_{6} \beta b
\end{array}\right] \sec h^{9} \alpha \xi \\
+8 \alpha^{4}\left[\begin{array}{l}
10\left(3 L_{3}+L_{6}\right) \beta a \\
-L_{5}\left(5 \gamma a^{2}+12 \beta a^{2}+4 \beta b^{2}\right)
\end{array}\right] \sec h^{10} \alpha \xi
\end{array}
$$

$+32 a^{2} \beta \alpha^{4} L_{5} \sec h^{12} \alpha \xi+2 \alpha c \gamma \sinh \alpha \xi \sec h^{3} \alpha \xi$ $+4 \alpha\left[\alpha\left(L_{2}+4 \alpha^{2}\left(L_{3}+L_{6}\right) \gamma \beta-\beta c\right)\right] \sinh \alpha \xi \sec h^{5} \alpha \xi$ $+4 \alpha^{2}\left[\begin{array}{c}8 \alpha^{2} L_{5} \gamma b a-3 \alpha^{2}\left(3 L_{3}+2 L_{6}\right) \gamma b \\ -2\left(L_{2}+20 \alpha^{2} L_{3}+16 \alpha^{2} L_{6}\right) \beta b\end{array}\right] \sinh \alpha \xi \sec h^{7} \alpha \xi$ $+16 \alpha^{4} L_{3} \gamma a \sinh \alpha \xi \sec h^{8} \alpha \xi$ $-32 \alpha^{4}\left(2 L_{3} \beta a+2 L_{5} \beta b a+L_{5} \gamma b a\right) \sinh \alpha \xi \sec h^{10} \alpha \xi$ $+96 \alpha^{4} L_{5} \beta a b \sinh \alpha \xi \sec h^{12} \alpha \xi$ $\left.+32 \alpha^{4} L_{5} \beta a b \sinh \alpha \xi \sec h^{12} \alpha \xi\right\}$

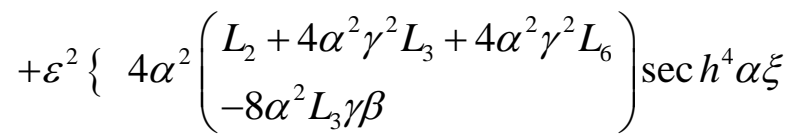
$+4 \alpha^{2}\left[\begin{array}{l}8 \alpha^{2} L_{5} \gamma^{2} a-\left(L_{2}+16 \alpha^{2} L_{3}+12 \alpha^{2} L_{6}\right) \gamma^{2} \\ -4\left(L_{2}+6 \alpha^{2} L_{3}+8 \alpha^{2} L_{6}\right) \beta \gamma\end{array}\right] \sec h^{6} \alpha \xi$ $+4 \alpha^{2}\left[\begin{array}{l}\left(4 L_{2}+64 \alpha^{2} L_{3}+64 \alpha^{2} L_{6}\right) \beta^{2} \\ +\left(9 \alpha^{2} L_{6}+12 \alpha^{2} L_{3}\right) \gamma^{2} \\ +4\left(L_{2}+29 \alpha^{2} L_{3}+22 \alpha^{2} L_{6}\right) \beta \gamma \\ -2 \alpha^{2} L_{5}\left(11 \gamma^{2}+24 \gamma \beta\right) a\end{array}\right] \sec h^{8} \alpha \xi$
$+4 \alpha^{2}\left[\begin{array}{l}2 \alpha^{2} L_{5}\left(60 \beta \gamma+7 \gamma^{2}+32 \beta^{2}\right) a \\ -4\left(L_{2}+40 \alpha^{2} L_{6}+46 \alpha^{2} L_{3}\right) \beta^{2} \\ -12 \alpha^{2}\left(7 L_{3}+5 L_{6}\right) \beta \gamma\end{array}\right] \sec h^{10} \alpha \xi$ $+16 \alpha^{4}\left[\begin{array}{l}\left(25 L_{6}+30 L_{3}\right) \beta^{2} \\ -\left(18 L_{5} \beta \gamma+16 L_{5} \beta^{2}\right) a\end{array}\right] \sec h^{12} \alpha \xi$ $+192 \alpha^{4} L_{5} \beta^{2} b \sec h^{13} \alpha \xi$ $+352 \alpha^{4} L_{5} \beta^{2} a \sec h^{14} \alpha \xi$ $-24 \alpha^{4} L_{5} \gamma^{2} b \sinh \alpha \xi \sec h^{7} \alpha \xi$ $+24 \alpha^{4} L_{5} \alpha^{4}\left(\gamma^{2}+4 \gamma \beta\right) \sinh \alpha \xi \sec h^{9} \alpha \xi$

$$
\begin{aligned}
& -160 \alpha^{4} L_{5} b\left(\beta \gamma+\beta^{2}\right) \sinh \alpha \xi \sec h^{11} \alpha \xi \\
& \left.+8 \alpha^{4} L_{5} b\left(4 \beta \gamma+\gamma^{2}\right) \sinh \alpha \xi \sec h^{14} \alpha \xi\right\} \\
& +\varepsilon^{3}\left\{-16 \alpha^{4} L_{5} \gamma^{3} \sec h^{6} \alpha \xi\right. \\
& +\alpha^{4} L_{5}\left(128 \beta \gamma^{2}+40 \gamma^{3}\right) \sec h^{8} \alpha \xi \\
& -8 \alpha^{4} L_{5}\left(40 \gamma \beta^{2}+38 \beta \gamma^{2}+3 \gamma^{3}\right) \sec h^{10} \alpha \xi \\
& +16 \alpha^{4} L_{5}\left(46 \beta^{2} \gamma+11 \beta \gamma^{2}+16 \beta^{3}\right) \sec h^{12} \alpha \xi \\
& -32 \alpha^{4} L_{5}\left(13 \beta^{2} \gamma+18 \beta^{3}\right) \sec h^{14} \alpha \xi \\
& \left.+320 \alpha^{4} L_{5} \beta^{3} \sec h^{16} \alpha \xi\right\}=0 \text {. }
\end{aligned}
$$

While identifying the different coefficients of $\sinh ^{i} \alpha \xi \sec h^{j} \alpha \xi$ where $i=0,1 \quad j=0,1,2, \ldots$ and according to the powers of $\varepsilon$ which is infinitely s mall, we get

Term in $\left(\varepsilon^{0}, \sec h^{2} \alpha \xi\right)$,

$$
c b+4 \alpha L_{2} a^{2}=0 .
$$

Term in $\left(\varepsilon^{0}, \sec h^{4} \alpha \xi\right)$,

$$
\left(L_{2}+\alpha^{2} L_{6}\right) b^{2}+4 \alpha^{2} L_{3} b^{2}-2 L_{1} a=0 .
$$

Term in $\left(\varepsilon^{0}, \sec h^{6} \alpha \xi\right)$

$2 \alpha^{2}\left(2 L_{5} a b^{2}-2 L_{5} a^{3}-L_{6} b^{2}\right)-2 a^{2} L_{2}-3 \alpha^{2} L_{3} b^{2}=0$.

Term in $\left(\varepsilon^{1}, \sec h^{2} \alpha \xi\right)$,

$$
L_{1}+4 \alpha^{2} L_{4}=0 \text {. }
$$

Term in $\left(\varepsilon^{1}, \sec h^{4} \alpha \xi\right)$,

$$
\begin{aligned}
& 3\left(L_{1}+20 \alpha^{2} L_{4}\right) \gamma+8\left(L_{1}+16 \alpha^{2} L_{4}\right) \beta \\
& -4\left(L_{2}+2 \alpha^{2} L_{3}\right) \gamma a-8 \alpha^{2} L_{5} a^{2} \gamma=0 .
\end{aligned}
$$

Term in $\left(\varepsilon^{1}, \sec h^{6} \alpha \xi\right)$,

$$
\begin{aligned}
& \alpha^{2} L_{5}\left(6 \gamma a^{2}+16 \beta a^{2}-3 \gamma b^{2}\right)+4\left(L_{2}+8 \alpha^{2} L_{3}\right) \beta a \\
& +2\left(L_{2}+8 \alpha^{2} L_{3}\right) \gamma a-5\left(L_{1}+52 \alpha^{2} L_{4}\right) \beta \\
& -30 \alpha^{2} L_{4} \gamma=0 .
\end{aligned}
$$

Term in $\left(\varepsilon^{1}, \sec h^{8} \alpha \xi\right)$,

$$
\begin{aligned}
& 420 \alpha^{2} L_{4} \beta a-8\left(L_{2}+23 \alpha^{2} L_{3}+4 \alpha^{2} L_{6}\right) \beta a \\
& -3 \alpha^{2}\left(2 L_{3}+L_{6}\right) \gamma a \\
& +\alpha^{2} L_{5}\left(16 \gamma a^{2}+16 \beta b^{2}+3 \gamma b^{2}\right)=0 .
\end{aligned}
$$


Term in $\left(\varepsilon^{1}, \sec h^{9} \alpha \xi\right)$,

$$
9 L_{3}-2 L_{5}(\gamma+2 \beta) b a+5 L_{6} \beta b=0 .
$$

Term in $\left(\varepsilon^{1}, \sec h^{10} \alpha \xi\right)$,

$$
\begin{aligned}
& 10\left(3 L_{3}+L_{6}\right) \beta a \\
& -L_{5}\left(5 \gamma a^{2}+12 \beta a^{2}+4 \beta b^{2}\right)=0 .
\end{aligned}
$$

The combination of the equations Eq.(15) and Eq.(16) gives a quadratic equation in $a$ of the form

$$
r a^{2}+s a+t=0
$$

where

$$
\begin{gathered}
r=4 \alpha^{2} L_{2} L_{5}+4 \alpha^{4} L_{5}\left(L_{6}+L_{3}\right), \\
s=2\left(L_{2}^{2}+\alpha^{2} L_{2} L_{6}+\alpha^{2} L_{2} L_{3}-4 \alpha^{2} L_{1} L_{5}\right), \\
t=4 \alpha^{2} L_{1} L_{6}+6 \alpha^{2} L_{1} L_{3} .
\end{gathered}
$$

The resolution of Eq.(17) requires a discussion around the parameters $r, s$ and $t$.

For $\quad r=0$ i.e. $\quad L_{2} /\left(L_{6}+L_{3}\right)=-\alpha^{2} \quad$ Eq. (17) becomes an equation of second order and admits the solution

$$
a=-\alpha^{2} L_{1}\left(2 L_{6}+3 L_{3}\right) /\left(\begin{array}{l}
L_{2}^{2}+\alpha^{2} L_{2}\left(L_{6}+L_{3}\right) \\
-4 \alpha^{2} L_{1} L_{5}
\end{array}\right)
$$

Inserting Eq.(18) in Eq.(9) we get

$$
b=\left\{-2 \alpha^{2} L_{1}^{2}\left(2 L_{6}+3 L_{3}\right) /\left(\left[\begin{array}{l}
{\left[L_{2}+\alpha^{2}\left(L_{6}+4 L_{3}\right)\right.} \\
\left.\left[\begin{array}{l}
L_{2}^{2}+\alpha^{2} L_{2}\left(L_{6}+L_{3}\right) \\
-4 \alpha^{2} L_{1} L_{5}
\end{array}\right]\right)
\end{array}\right)\right\}^{\frac{1}{2}}\right.
$$

For $r \neq 0$ i.e. $L_{2} /\left(L_{6}+L_{3}\right) \neq-\alpha^{2}$, Eq.(17) is of second order in $a$. The resolution of Eq.(17) in these conditions gives:
For $\Delta^{\prime} \geq 0$, we obtain the solution

$$
a=\left(-\frac{s}{2} \pm \sqrt{\Delta^{\prime}}\right) / r,
$$

and

$b=\left[2 L_{1}\left(-\frac{s}{2} \pm \sqrt{\Delta^{\prime}}\right) /\left(L_{2}+\alpha^{2} L_{6}+4 \alpha^{2} L_{3}\right) r\right]^{\frac{1}{2}}$

where $\Delta^{\prime}=\left(s^{2} / 4\right)-r t$.

- For $\Delta^{\prime} \prec 0$, we obtain the following roots

$$
a=\left(-\frac{s}{2} \pm i \sqrt{-\Delta^{\prime}}\right) / r
$$

and

$$
b=\left[2 L_{1}\left(-\frac{s}{2} \pm i \sqrt{-\Delta^{\prime}}\right) /\left(L_{2}+\alpha^{2} L_{6}+4 \alpha^{2} L_{3}\right) r\right]^{\frac{1}{2}} \text {. }
$$

On the other hand, while co mbin ing equations Eq.(15) and Eq.(16), one gets $\beta$ and $\gamma$ as functions of $a$ and $b$

$$
\beta=45 L_{3} a /\left(\begin{array}{l}
60 L_{3} a-5 L_{6} a \\
-4 L_{5} a^{2}-8 L_{5} b^{2}
\end{array}\right) b,
$$

and

$$
\gamma=\left[\begin{array}{l}
\left(270 L_{3}^{2}+90 L_{6} L_{3}\right) a \\
-108 L_{3} L_{5} a^{2} \\
-36 L_{5} L_{3} b^{2}
\end{array}\right] /\left[\begin{array}{l}
\left(60 L_{3} L_{5}-5 L_{6} L_{5}\right) a^{2} b \\
-4 L_{5}^{2} a^{3} b-8 L_{5}^{2} a b^{3}
\end{array}\right]
$$

where $a$ and $b$ are values given respectively by equations Eq.(18), Eq.(19), Eq.(20), Eq.(21), Eq.(22) and Eq.(23).

Taking into account equations Eq.(18), Eq. (19),..., Eq. (25) in Eq. (6) we get three great families of solutions as seen below.

For $r=0$, i.e. $L_{2} /\left(L_{6}+L_{3}\right)=-\alpha^{2}$, the first family is given by

$$
\begin{gathered}
\psi(\xi)=\left[-\alpha^{2} L_{1}\left(2 L_{6}+3 L_{3}\right) / L_{2}^{2}+\alpha^{2} L_{2}\left(L_{6}+L_{3}\right)-4 \alpha^{2} L_{1} L_{5}\right] \sec h^{2} \alpha \xi \\
+\left\{-2 \alpha^{2} L_{1}^{2}\left(2 L_{6}+3 L_{3}\right) /\left[L_{2}+\alpha^{2}\left(L_{6}+4 L_{3}\right)\right]\left[L_{2}^{2}+\alpha^{2} L_{2}\left(L_{6}+L_{3}\right)-4 \alpha^{2} L_{1} L_{5}\right]\right\}^{\frac{1}{2}} \tanh h \alpha \xi \\
+\varepsilon\left[\begin{array}{l}
\left.\begin{array}{l}
\left.45 L_{3} a /\left(60 L_{3} a-5 L_{6} a-4 L_{5} a^{2}-8 L_{5} b^{2}\right) b\right] \sec h^{4} \alpha \xi \\
{\left[\left(270 L_{3}^{2}+90 L_{6} L_{3}\right) a-108 L_{3} L_{5} a^{2}-36 L_{5} L_{3} b^{2}\right]} \\
+\left[\left(60 L_{3} L_{5}-5 L_{6} L_{5}\right) a^{2} b-4 L_{5}^{2} a^{3} b-8 L_{5}^{2} a b^{3}\right]
\end{array}\right\} \tanh ^{2} \alpha \xi
\end{array}\right],
\end{gathered}
$$

where $a$ and $b$ are given by Eq.(18) and Eq.(19).

For $r \neq 0$, i.e. $L_{2} /\left(L_{6}+L_{3}\right) \neq-\alpha^{2}$, we obtain the last two families of solutions 


$$
\begin{gathered}
\psi(\xi)=\left[\left(-\frac{s}{2} \pm \sqrt{\Delta^{\prime}}\right) / r\right] \sec h^{2} \alpha \xi+\left[2 L_{1}\left(-\frac{s}{2} \pm \sqrt{\Delta^{\prime}}\right) /\left(L_{2}+\alpha^{2} L_{6}+4 \alpha^{2} L_{3}\right) r\right]^{\frac{1}{2}} \tanh \alpha \xi \\
+\varepsilon\left\{\begin{array}{l}
\left.45 L_{3} a /\left(60 L_{3} a-5 L_{6} a-4 L_{5} a^{2}-8 L_{5} b^{2}\right) b\right] \sec h^{4} \alpha \xi \\
+\left\{\begin{array}{l}
{\left[\left(270 L_{3}^{2}+90 L_{6} L_{3}\right) a-108 L_{3} L_{5} a^{2}-36 L_{5} L_{3} b^{2}\right]} \\
/\left[\left(60 L_{3} L_{5}-5 L_{6} L_{5}\right) a^{2} b-4 L_{5}^{2} a^{3} b-8 L_{5}^{2} a b^{3}\right]
\end{array}\right\} \tanh ^{2} \alpha \xi
\end{array}\right\},
\end{gathered}
$$

where $\Delta^{\prime} \geq 0, a$ and $b$ given by Eq.(22) and Eq.(23). We also have

$$
\begin{aligned}
& \psi(\xi)=\left[\left(-\frac{s}{2} \pm i \sqrt{-\Delta^{\prime}}\right) / r\right] \sec h^{2} \alpha \xi+\left[2 L_{1}\left(-\frac{s}{2} \pm i \sqrt{-\Delta^{\prime}}\right) /\left(L_{2}+\alpha^{2} L_{6}+4 \alpha^{2} L_{3}\right) r\right]^{\frac{1}{2}} \tanh \alpha \xi \\
& +\varepsilon\left\{\begin{array}{l}
{\left[45 L_{3} a /\left(60 L_{3} a-5 L_{6} a-4 L_{5} a^{2}-8 L_{5} b^{2}\right) b\right] \sec h^{4} \alpha \xi} \\
+\left\{\begin{array}{l}
{\left[\left(270 L_{3}^{2}+90 L_{6} L_{3}\right) a-108 L_{3} L_{5} a^{2}-36 L_{5} L_{3} b^{2}\right]} \\
/\left[\left(60 L_{3} L_{5}-5 L_{6} L_{5}\right) a^{2} b-4 L_{5}^{2} a^{3} b-8 L_{5}^{2} a b^{3}\right]
\end{array}\right\} \tanh ^{2} \alpha \xi
\end{array}\right\},
\end{aligned}
$$

where $\Delta^{\prime} \prec 0, \quad a$ and $b$ are given by Eq.(22) and Eq.(23).

To better understand the notion of dominant and less dominant parts of wave $\psi=a \sec h^{2} \alpha \xi+b \tanh \alpha \xi+\varepsilon\left(\beta \sec h^{4} \alpha \xi+\gamma \tanh ^{2} \alpha \xi\right)$, we are engaged in the representation of the two main parts that constitute $\psi$. If we state that $\psi_{1}=a \sec h^{2} \alpha \xi+b \tanh \alpha \xi$ and $\psi_{2}=\beta \sec h^{4} \alpha \xi+\gamma \tanh ^{2} \alpha \xi$, the wave becomes $\psi=\psi_{1}+\varepsilon \psi_{2}$. Figure 1 shows the representation of $\psi_{1}$, which is the profile of a soliton wave of a kin k type. Figure 2 and Figure 3 show the representation of $\psi_{2}$ for a few values of $\beta$ and $\gamma$. The two profiles obtained in Figure 2 and Figure 3 are soliton waves of pulse nature. Figure 4 gives the representation of solution in general $\psi=\psi_{1}+\varepsilon \psi_{2}$ for $\varepsilon$ very small ( $\varepsilon=0.01$ ). The profile obtained is that of a kink. We simply realize that $\psi$ can take the form of a kink or the form of a pulse depending on the value of $\varepsilon$. As regard our research work, we have chosen for our dominant part $\psi_{1}$. The practical interpretation that we can give to this solution is that of a soliton wave solution which changes its form with respect to the environment in which it happens to be or to the obstacle to overcome in its medium of propagation. In the example considered hereafter the soliton $\psi$ can lose its kink or pulse form depending on the conditions under which it is subjected.

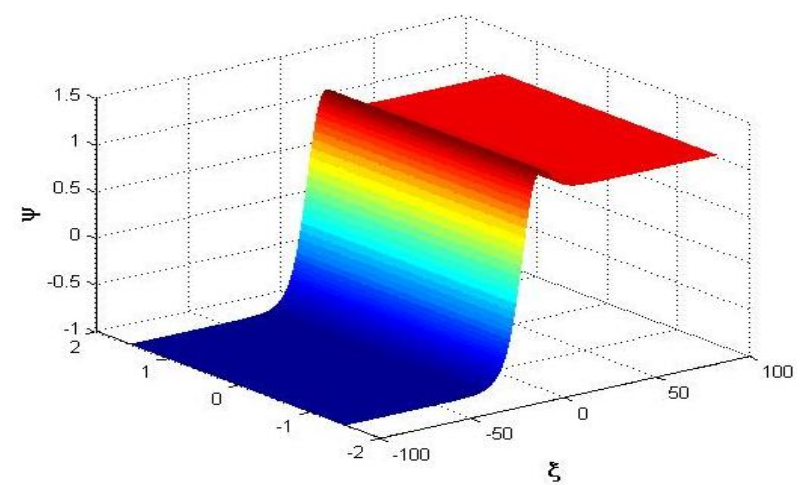

Figure 1. Representative curve of the soliton profile of the solution $\psi_{1}=a \sec h^{2} \alpha \xi+b \tanh \alpha \xi$, for $a=1, b=1, \alpha=0.1$.

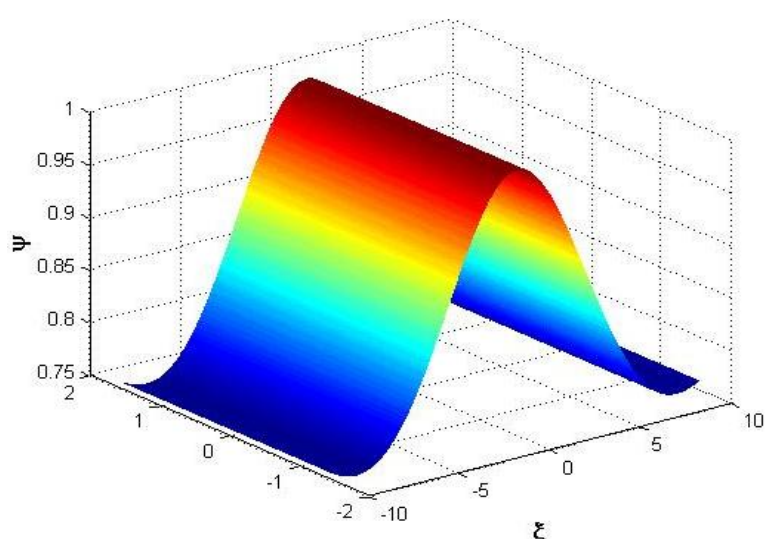

Figure 2. Representative curve of the soliton profile of $\psi_{2}=\beta \sec ^{4} \alpha \xi+\gamma \tanh ^{2} \alpha \xi$, for $a=1, b=1, \alpha=0.1$. and and in the case where $\xi \in[-100,100], \psi \in[-1.5,1.5]$ 


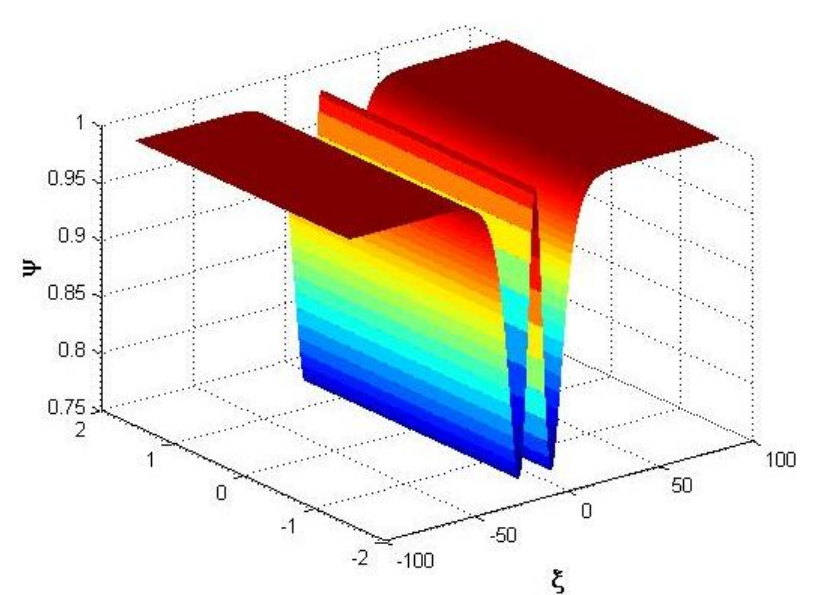

Figure 3. Representative curve of the soliton profile of $\psi_{2}=\beta \sec h^{4} \alpha \xi+\gamma \tanh ^{2} \alpha \xi \quad$, for $a=1, b=1$, $\alpha=0.1$ and in the case where $\xi \in[-10,10], \psi \in[-1.5,1.5]$

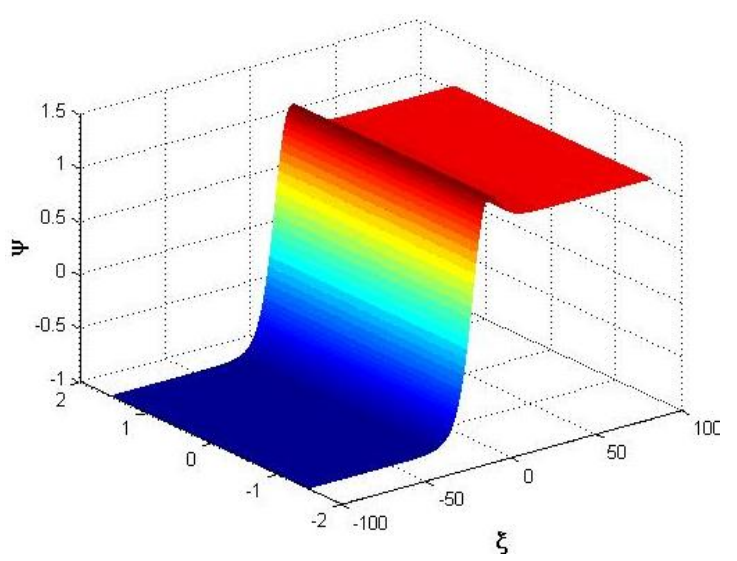

Figure 4. Representative curve of the soliton profile of the combined solution $\psi=a \sec h^{2} \alpha \xi+b \tanh \alpha \xi+\varepsilon\left(\beta \sec h^{4} \alpha \xi+\gamma \tanh ^{2} \alpha \xi\right)$, $a=1, b=1, \alpha=0.1, \beta=1, \gamma=1$ and $\varepsilon=0.01$

\section{Conclusions}

We have just polished up this work by putting forward a solution to Kuramoto-Sivashinsky's equation in the form of a combine soliton wave. In the course of our work we have made up a solution of the form $\psi(\xi)=\psi_{1}(\xi)+\varepsilon \psi_{2}(\xi)$, where $\psi_{1}$ and $\psi_{2}$ are solitary waves. Through the BDK innovative method mentioned above, we want to prove that it is possible to make up modulated soliton solutions of the form $\psi=\psi_{1}(\xi)+\varepsilon \psi_{2}(\xi)+\varepsilon^{2} \psi_{3}(\xi)+\ldots+\varepsilon^{n} \psi_{n}$, where $\psi_{1}, \psi_{2}, \ldots, \psi_{n}$ represent solitary waves. This form that we are putting forward as regard all calculations and analysis undertaken up to date, helps us to confirm our satisfaction as regard the usage of the BDK method in making up modulated soliton solution of Kuramoto-Sivashinsky's equation.

On the whole, projecting our reasoning rays beyond its limit, multi-soliton put into evidence experimentally will be an object of attraction for all scientists.

\section{ACKNOWLEDGEMENTS}

This work is supported by the Ministry of higher Education of Cameroon through its project of support to the scientific research.

\section{REFERENCES}

[1] Y. Kuramoto, Chemical oscillations, waves and turbulence, Springer, B erlin, 1984.

[2] G. Sivashinsky, On flame propagation under conditions of stoichiometry, SIAM Journal of Applied Mathematics, Vol.39, pp. 67-82, 1980.

[3] G. I. Sivashinsky, Instabilities, pattern formation and turbulence in flames, Annual Review of Fluid Mechanics, Vol. 15, pp. 179-183, 1983.

[4] G. I. Sivashinsky, D. M. Michelson, On irregular wary flow of a liquid film down a vertical plane, Progress of Theory Physics, Phys (Progress Lett.), Vol.63, pp. 2112-2114, 1980.

[5] G. P. Agrawal, Nonlinear fiber Optics, $2^{\text {nd }}$ edition. Academic Press Inc. San Diego, 1995.

[6] J. H. B. Nijhof, N. J. Doran, W. Fory siak, F. M. Knox, Stable Soliton-like propagation in dispersion managed systems with net anomalous, zero and normal dispersion, Electronics Letters, Vol. 33, pp.1726-1727.

[7] V. E. Zakharov, S. Wabnitz. Optical Solitons: Theoretical challenges and industrial perspectives: Springer- Verlag, 1998.

[8] A. M. Wazwaz, the tanh method: Exact solutions of the sine-Gordon and the sinh-Gordon equations, Applied Mathematics and Computation Vol. 167, pp. 1196-1210, 2005.

[9] A. M. Wazwaz, Partial differential equations: Methods and applications, Balkema, the Netherlands, 2002

[10] A. M. Wazwaz, Compactons dispersive structures for variants of the $K(n, n)$ and the $K P$ equations, Chaos Solitons, \&Fractals, Vol.13, pp. 321-330, 2002.

[11] A. M. Wazwaz, Exact specific solutions with solitary patterns for the nonlinear dispersive $K(m, n)$ equations, Chaos, Solitons \&Fractals, Vol.13, pp.161-170, 2001.

[12] A. M. Wazwaz, The tanh method for traveling wave solutions of nonlinear equations, Applied Mathematics and Computation, Vol. 133, pp. 213-227, 2002.

[13] A. M. Wazwaz, A study of nonlinear dispersive equations with solitary wave solutions having compact support, Mathematics and Computers in Simulation, Vol.56, pp. 269-276, 2001.

[14] H. Stendel , D. J. Kaup, Inverse scattering method applied to degenerate two-photon propagation in the law excitation limit, Journal of Phy sics A: Mathematical and Theoretical, Vol.33, pp. 1445-1457, 2000.

[15] R. A. Fisher, W. K. Bischel, The role of linear dispersion in 
plane-wave self phase modulation, Journal of Applied Physics Letters, Vol. 23, pp. 661-663, 1973.

[16] D. J. Kaup, T. I. Lakoba, Variational method: How it can generate false instabilities, Journal of Mathematical Physics, Vol.37, pp. 3442-3462, 1996.

[17] D. J. Kaup, T. I. Lakoba, The squared eigenfunctions of the massive Thirring model in laboratory coordinates, Journal of Mathematical Physics, Vol. 37, pp. 308-323, 1996.

[18] Qi Wang, Chen Yong, Hongqinq Zhang, A new jacobi elliptic function rational expansion method and its application to (1+1)-dimensional dispersive long wave equation, Chaos, Solitons \& Fractals, Vol. 23, pp. 477-483, 2005.

[19] He Ji Huan, Some asymptotic method for strongly nonlinear equations, International Journal of modern phy sics B, Vol. 20, pp. 1141-1199, 2006.

[20] J. R. Bogning, T. C. Kofane, Analytical solutions of the discrete nonlinear Schrödinger equation in arrays of optical fibers Chaos, Solitons \& Fractals, Vol. 28, pp. 148-153, 2006.

[21] D. J. Kaup, T. I. Lakoba, Y. Matsuno, Perturbation theory for the Benjamin-Ono equation, Inverse Problems Vol.15, pp. 215-240, 1999.

[22] E. Fan, extended tanh-function method and its applications to nonlinear equations, Phy sics Letters A, Vol. 277, pp. 212-218, 2000.

[23] E. Fan, Y. C. Hon, Applications of extended tanh method to "special" types of nonlinear equations, App lied Mathematics and Computation, Vol. 141, pp. 351-358, 2003.

[24] A. M. Wazwaz, the tanh-coth and the sine-cosine methods for kinks, solitons, and periodic solutions for the pochhammer-chree equations, Applied Mathematics and Computation, Vol.195, pp. 24-33, 2008.

[25] E. Fan, C. H. Zhang, A note on the homogeneous balance method, physics Letters A, Vol. 246, pp. 403-406, 1998.

[26] E. Fan, Two new applications of the homogeneous balance method, phy sics Letters A, Vol. 265, pp. 353-357, 2000.
[27] J. H. He, X. H. Wu, Exp-function method for nonlinear wave equations, Chaos ,solitons \&Fractals , Vol.30, pp. 700-708, 2006.

[28] J. H. He, M. A. Abdou, New periodic solutions for nonlinear evolution equations using Exp-function method, Chaos, solitons \& Fractal Vol. 34, pp. 1421-1429, 2007.

[29] Xi-jun deng, periodic and solitary wave solutions in quadratic nonlinear media, Chinese Journal of Physics, Vol. 46, pp. 511-516, 2008

[30] W. Malfiet, the tanh method I: Exact solutions of nonlinear evolution and wave equations, American Journal of Physics, Vol. 4, pp. 650-654, 1992.

[31] A. M. Wazwaz, compactons and solitary patterns structures for variants of the $\mathrm{KdV}$ and the $\mathrm{KP}$ equations, Applied Mathematics and Computation, Vol. 139, pp. 37-54, 2006

[32] L. Ji, C. X. Ping, Y. L. Jun, Soliton and periodic traveling wave solutions for quadratic nonlinear media, Chinese Physics Letters, Vol. 23, pp.147-150, 2006.

[33] Clovis T Djeumen Tchaho, Jean R Bogning, Timoleon C Kofane, Construction of the analy tical solitary wave solutions of modified Kuramoto-Sivashinsky equation by the method of identification of coefficients of the hyperbolic functions, Far East Journal of Dynamical Systems, Vol. 14, pp. 17-34, 2010.

[34] Clovis T Djeumen Tchaho, Jean R Bogning, Timoleon C Kofane, Multi-Soliton solutions of the modified Kuramoto-Sivashinsky's equation by the BDK method, Far East Journal of Dynamical Systems, Vol. 15, pp. 15(2), pp.83-98, 20011.

[35] Jean R Bogning, Clovis T Djeumen Tchaho and Timoléon C Kofané. Construction of the soliton solutions of the GinzburgLandau equations by the Bogning-Djeumen Tchaho-Kofané method, Physica Scripta, Vol. 85, pp. 025013-025018, 2012.

[36] Jean R Bogning, Clovis $\mathrm{T}$ Djeumen Tchaho, Timoléon C Kofane, Generalization of the Bogning- Djeumen Tchaho-Kofane Method for the construction of the solitary waves and the survey of the instabilities, Far East Journal of Dynamical Sy stems 2012 ( submitted) 